\title{
Servais Paul (coord.) : L'évaluation de la recherche en sciences humaines et sociales. Regards de chercheurs
} Joachim Schöpfel et Chérifa Boukacem-Zeghmouri

\section{(2) OpenEdition}

\section{Journals}

Édition électronique

URL : http://journals.openedition.org/edc/3231

DOI : $10.4000 /$ edc.3231

ISSN : 2101-0366

Éditeur

Université de Lille

Édition imprimée

Date de publication : 1 décembre 2011

Pagination : 201-204

ISBN : 978-2-917562-06-2

ISSN : $1270-6841$

Référence électronique

Joachim Schöpfel et Chérifa Boukacem-Zeghmouri, « Servais Paul (coord.) : L'évaluation de la recherche en sciences humaines et sociales. Regards de chercheurs », Études de communication [En ligne], 37 |

2011, mis en ligne le 07 mars 2012, consulté le 22 septembre 2020. URL : http://

journals.openedition.org/edc/3231; DOI : https://doi.org/10.4000/edc.3231

Ce document a été généré automatiquement le 22 septembre 2020.

(c) Tous droits réservés 


\title{
Servais Paul (coord.) : L'évaluation de la recherche en sciences humaines et sociales. Regards de chercheurs
}

\author{
Joachim Schöpfel et Chérifa Boukacem-Zeghmouri
}

\section{RÉFÉRENCE}

Servais Paul (coord.) : L'évaluation de la recherche en sciences humaines et sociales. Regards de chercheurs, Louvain-la-Neuve, Bruylant-Academia, 2011, 298 p. - (Collection Intellection $n^{\circ}$ 14), ISBN $9782806100238,35 €$.

1 L'évaluation des établissements, laboratoires, chercheurs et formations est une réalité qu'on peut apprécier ou non mais qu'il convient d'abord de connaître et comprendre. L'Institut d'Analyse du Changement dans l'Histoire et les Sociétés Contemporaines (IACCHOS) ${ }^{1}$ de l'Université Catholique de Louvain avait organisé en $2009^{2}$ un colloque pour analyser l'évaluation de la recherche des points de vue politiques, historiques et méthodologiques.

2 Les questions : «Qu'est-ce que évaluer la recherche ? Pourquoi et pour quoi évaluer la recherche? Comment évaluer la recherche ? Y a-t-il des pièges à l'évaluation et lesquels? La transition de l'évaluation de la recherche à l'évaluation des chercheurs est-elle inévitable, voire souhaitable ? L'évaluation induit-elle des changements dans la recherche?».

Le but était de confronter des expériences et pratiques culturelles et nationales diversifiées, en limitant le champ d'investigation aux sciences humaines et sociales.

Un peu plus d'un an après le colloque, Bruylant-Academia (Louvain-la-Neuve) vient de publier l'essentiel des actes sous forme d'une monographie aussi intéressante que riche et instructive. Voici un bref aperçu du contenu ${ }^{3}$. 
5 L'ouvrage contient treize contributions, introduites par une courte préface de Paul Servais, l'actuel directeur de l'IACCHOS, qui revient sur l'objectif de ce travail collaboratif, qui consiste à questionner l'évaluation, en tant que symptôme d'une transformation des relations entre « connaissances » et « politiques ».

6 Les textes sont répartis sur trois parties. La première partie - Enjeux - regroupe trois textes sur 70 pages. Sans remettre en question le principe de l'évaluation, les auteurs expliquent comment sa mise en œuvre ne prend en compte ni la spécificité de l'activité de recherche du chercheur, ni sa pluralité, et encore moins celle de la spécificité de l'établissement dans laquelle elle se déroule. Il en découle un sentiment de découragement, d'impuissance, de scepticisme partagé entre chercheurs dont l'un des effets pervers serait d'engendrer une « recherche bibliométrisée » (Eraly, p. 27). Le ton caustique cède parfois le pas à un ton alarmiste, qui met en garde contre une évaluation qui repose sur des procédures empruntées à la Gestion et à la Qualité, faisant ainsi le déni de la "réalité du travail de recherche ». Ce qui peut conduire à « des fictions de qualité détachées des pratiques effectives d'enseignement et de recherche » (Périlleux, p. 48). Et on s'étonne que la fascination pour le modèle étasunien fasse perdre de vue qu'en adoptant l'évaluation, dans ses formes actuelles, on importait un modèle libéral dans un contexte républicain, en contradiction avec l'idée d'Université, service national (La Combe, pp. 78-79).

7 La deuxième partie - Mises en œuvres - constitue le cœur de l'ouvrage, avec six textes sur 130 pages. Elle insiste sur le fait que l'évaluation est une pratique ancienne et plurielle dans l'Université (Béaur, p. 167). Elle montre aussi comment des pays et des contextes différents se sont saisis de l'évaluation, l'ont affrontée, adoptée et adaptée à leurs structures, à leurs organisations, en tenant compte de ce qui fait la base: la communauté savante. Cela témoigne d'une «transformation profonde de la place du chercheur qui est de moins en moins un atome libre et de plus en plus le membre d'une équipe structurée» (Martin, p.153). L'impact sociétal des recherches est particulièrement souligné dans la démarche d'évaluation canadienne (Belin, p. 89) ; il vient illustrer la spécificité des objets et des méthodes de recherche SHS qui se retrouvent en porte à faux vis-à-vis des méthodes et des indicateurs d'évaluation qu'on leur impose (Halloin, p. 109), (De Ketele, p. 183). Par ailleurs, la place de l'enseignement supérieur dans la démarche de l'évaluation est particulièrement soulignée par la tradition américaine (Smith, p. 159).

8 La troisième et dernière partie - Critiques - réunit quatre communications sur 75 pages. On comprend comment, au sein des comités d'évaluation, l'évaluation se construit avec des critères objectifs et d'autres moins objectifs, liés à la nature fondamentalement humaine des évaluateurs (Lamont, p. 213). On comprend également comment au sein de ces comités, les recherches qualitatives peuvent être difficiles à appréhender par des processus standardisés d'évaluation, surtout en regard d'évaluateurs issus des sciences dures (De Sradan, p. 231). Le classement des revues en SHS est questionné par le biais des «présupposés de l'évaluation qu'il est censé servir » (Galvez-Behar, p. 254). Enfin, la synthèse des travaux menés par Yves Gingras met l'accent sur les nuances significatives qui existent entre sciences sociales et sciences humaines; ce qui appelle à une plus grande prudence dans la mise en œuvre d'une politique d'évaluation appliquée au bloc trop souvent considéré comme homogène des SHS (Du Roy, p. 267).

9 Qui sont les 16 auteurs? Il s'agit surtout d'historiens mais aussi de sociologues, psychologues et représentants d'organismes scientifiques (agences de moyens). Ils 
travaillent en Belgique, France, Canada et aux États-Unis. Parmi les organismes d'affiliation on trouve l'Université Catholique de Louvain et d'autres universités (Nancy, Lille, Harvard, Pennsylvanie, Philadelphie), le CNRS et la FSR belge.

Quelques textes se détachent nettement, par leur spécificité. Celui d'Alain Eraly traduit bien la perplexité des communautés savantes vis-à-vis de politiques d'évaluation ayant du mal à préciser leurs objectifs. Le texte de Pierre Judet de La Combe montre comment la complexité et l'historicité des institutions - françaises en l'occurrence - sont ignorées par les politiques d'évaluation. Le texte de Morgane Belin et Corentin de Favreau montre comment le modèle canadien tente de trouver un juste milieu entre la nécessité d'évaluer et l'importance de prendre en compte les spécificités de la recherche et du chercheur dans une dynamique constructive. Enfin, les textes de JeanMarie de Ketele, Jean-Pierre Olivier de Sradan et la synthèse de Gaëtan du Roy et Caroline Sappia donnent la mesure de la différence entre les domaines (SHS et STM), mais aussi entre les disciplines, au sein des SHS.

11 Les textes sont écrits avec une démarche pédagogique - montrer, expliquer, faire comprendre avant de porter un jugement et/ou de condamner. L'essentiel des textes est basé sur la réflexion qui pousse à la discussion et ne repose donc pas sur des études empiriques ou extensives.

12 Le style est généralement lisible et clair. Le lecteur est pris au sérieux dans la mesure où on lui donne l'occasion d'apprendre, de comparer, d'analyser en changeant l'approche et l'environnement, à la place de lui dire ce qu'il doit penser.

13 A qui s'adresse ce livre ? Tout d'abord aux chercheurs eux-mêmes, en première ligne et directement concernés par le dispositif d'évaluation. Puis aux doctorants et étudiants en master de recherche qui s'orientent vers une carrière scientifique. Mais le livre sera également fort utile entre les mains des gestionnaires d'établissements et de laboratoires et des professionnels de l'information qui d'une façon ou d'une autre, sont parties prenantes et acteurs de l'évaluation. Il les aidera à prendre du recul et à mieux comprendre les enjeux, problèmes et perspectives du système en place.

(Tous les sites ont été consultés entre le 20 juin et le 26 septembre 2011).

\section{NOTES}

1. http://www.uclouvain.be/iacchos.html

2. http://calenda.revues.org/nouvelle14911.html

3. Le sommaire du livre est en ligne sur le site de l'éditeur : http://www.academia-bruylant.be/ pdf/20023.pdf 


\section{AUTEURS}

JOACHIM SCHÖPFEL

Maître de conférences en Sciences de l'Information et de la Communication Laboratoire GERiiCO (EA 4073) - Université de Lille 3 joachim.schopfel@univ-lille3.fr

\section{CHÉRIFA BOUKACEM-ZEGHMOURI}

Maître de conférences en Sciences de l'Information et de la Communication URFIST de Lyon boukacemc@yahoo.fr 